\title{
Ranked Sparse Signal Support Detection
}

\author{
Alyson K. Fletcher, Member, IEEE, Sundeep Rangan, Member, IEEE, and Vivek K Goyal, Senior Member, IEEE
}

\begin{abstract}
This paper considers the problem of detecting the support (sparsity pattern) of a sparse vector from random noisy measurements. Conditional power of a component of the sparse vector is defined as the energy conditioned on the component being nonzero. Analysis of a simplified version of orthogonal matching pursuit (OMP) called sequential OMP (SequOMP) demonstrates the importance of knowledge of the rankings of conditional powers. When the simple SequOMP algorithm is applied to components in nonincreasing order of conditional power, the detrimental effect of dynamic range on thresholding performance is eliminated. Furthermore, under the most favorable conditional powers, the performance of SequOMP approaches maximum likelihood performance at high signal-to-noise ratio.
\end{abstract}

Index Terms-Compressed sensing, convex optimization, lasso, maximum likelihood estimation, orthogonal matching pursuit, random matrices, sparse Bayesian learning, sparsity, thresholding.

\section{INTRODUCTION}

$\mathbf{S}$ ETS of signals that are sparse or approximately sparse with respect to some basis are ubiquitous because signal modeling often has the implicit goal of finding such bases. Using a sparsifying basis, a simple abstraction that applies in many settings is for

$$
\mathbf{y}=\mathbf{A x}+\mathbf{d}
$$

to be observed, where $\mathbf{A} \in \mathbb{R}^{m \times n}$ is known, $\mathbf{x} \in \mathbb{R}^{n}$ is the unknown sparse signal of interest, and $\mathbf{d} \in \mathbb{R}^{m}$ is random noise. When $m<n$, constraints or prior information about $\mathbf{x}$ are essential to both estimation (finding vector $\hat{\mathbf{x}}(\mathbf{y})$ such that $\|\mathbf{x}-\hat{\mathbf{x}}\|$ is small) and detection (finding index set $\hat{I}(\mathbf{y})$ equal to the support of $\mathbf{x}$ ). The focus of this paper is on the use of magnitude rank information on $\mathbf{x}$ - in addition to sparsity - in the support detection problem. We show that certain scaling laws relating the problem dimensions and the noise level are changed dramatically by exploiting the rank information in a simple sequential detection algorithm.

The simplicity of the observation model (1) belies the variety of questions that can be posed and the difficulty of precise analysis. In general, the performance of any algorithm is a complicated function of $\mathbf{A}, \mathbf{x}$, and the distribution of $\mathbf{d}$. To enable

Manuscript received November 02, 2011; revised April 24, 2012; accepted June 18, 2012. Date of publication July 16, 2012; date of current version October 09,2012 . The associate editor coordinating the review of this manuscript and approving it for publication was Prof. Namrata Vaswani. The work of V. $\mathrm{K}$ Goyal was supported in part by the National Science Foundation under CAREER Grant No. 0643836. This work was presented in part at the IEEE International Symposium on Information Theory, Seoul, Korea, June-July 2009

A. K. Fletcher is with the Department of Electrical Engineering, University of California, Santa Cruz, CA 95064 USA (e-mail: afletcher@soe.ucsc.edu).

S. Rangan is with the Department of Electrical and Computer Engineering, Polytechnic Institute of New York University, Brooklyn, NY 11201 USA (e-mail: srangan@poly.edu).

V.K Goyal is with the Research Laboratory of Electronics, Massachusetts Institute of Technology, Cambridge, MA 02139 USA (e-mail: vgoyal@mit.edu).

Digital Object Identifier 10.1109/TSP.2012.2208957 results that show the qualitative behavior in terms of problem dimensions and a few other parameters, we assume the entries of $\mathbf{A}$ are i.i.d. normal, and we describe $\mathbf{x}$ by its energy and its smallest-magnitude nonzero entry.

We consider a partially-random signal model

$$
x_{j}=b_{j} s_{j}, \quad j=1,2, \ldots, n,
$$

where components of vector $\mathbf{b}$ are i.i.d. Bernoulli random variables with $\operatorname{Pr}\left(b_{j}=1\right)=1-\operatorname{Pr}\left(b_{j}=0\right)=\lambda>0$ and $\mathbf{s}$ is a nonrandom parameter vector with all nonzero entries. The value $s_{j}^{2}$ represents the conditional power of the component $x_{j}$ in the event that $b_{j}=1$. We consider the problem where the estimator knows neither $b_{j}$ nor $s_{j}$, but may know the order or rank of the conditional powers. In this case, the estimator can, for example, sort the components of $\mathbf{s}$ in an order such that

$$
\left|s_{1}\right| \geq\left|s_{2}\right| \geq \cdots \geq\left|s_{n}\right|>0 .
$$

A stylized application in which the conditional ranks (and furthermore approximate conditional powers) can be known is random access communication as described in [1]. Also, partial orders of conditional powers can be known in some applications because of the magnitude variation of wavelet coefficients across scale [2]. Along with being motivated by these applications, we aim to provide a new theoretical grounding for a known empirical phenomenon: orthogonal matching pursuit (OMP) and sparse Bayesian learning (see references below) exhibit improvements in detection performance when the nonzero entries of the signal have higher dynamic range.

\section{A. Main Contribution}

Rank information is extremely valuable in support detection. Abstracting from the applications above, we show that when conditional rank information is available, a very simple detector, termed sequential orthogonal matching pursuit (SequOMP), can be effective. The SequOMP algorithm is a one-pass version of the well-known OMP algorithm. Similar to several works in sparsity pattern recovery [3]-[5], we analyze the performance of SequOMP by estimating a scaling on the minimum number of measurements $m$ to asymptotically reliably detect the sparsity pattern (support) of $\mathbf{x}$ in the limit of large random matrices A. Although the SequOMP algorithm is extremely simple, we show:

- When the power orders are known and the signal-to-noise ratio (SNR) is high, the SequOMP algorithm exhibits a scaling in the minimum number of measurements for sparsity pattern recovery that is within a constant factor of the more sophisticated lasso and OMP algorithms. In particular, SequOMP exhibits a resistance to large dynamic ranges, which is one of the main motivations for using lasso and OMP.

- When the power profile can be optimized, SequOMP can achieve measurement scaling for sparsity pattern recovery 
TABLE I

Summary of Results on Measurement Scalings for Asymptotic Reliable Detection for Various Detection Algorithms. Only Leading Terms Are Shown. See Body for Definitions and Technical Limitations

\begin{tabular}{|c|c|c|}
\hline & finite SNR - MAR & SNR $\cdot$ MAR $\rightarrow \infty$ \\
\hline Necessary for ML & $\begin{array}{l}m>\frac{2}{\text { MAR.SNR }} k \log (n-k) \\
\text { Fletcher et al. [5, Thm. 1] }\end{array}$ & $\begin{array}{c}m>k \\
\text { (elementary) }\end{array}$ \\
\hline Sufficient for ML & $\begin{array}{c}m>\frac{C}{\text { MAR } \cdot \text { SNR }} k \log (n-k) \\
\text { Wainwright [3] }\end{array}$ & $\begin{array}{c}m>k \\
\text { (elementary) }\end{array}$ \\
\hline $\begin{array}{l}\text { Sufficient for SequOMP } \\
\text { with best power profile }\end{array}$ & $\begin{array}{c}m>\frac{8}{\log (1+\mathrm{SNR})} k \log (n-k) \\
\text { From Theorem } 1 \text { (Section IV-D) }\end{array}$ & $\begin{array}{c}\qquad m>9 k \\
\text { From Theorem } \mathbf{1} \text { (Section IV-E) }\end{array}$ \\
\hline $\begin{array}{c}\text { Sufficient for SequOMP } \\
\text { with known conditional ranks }\end{array}$ & $\begin{array}{c}m>\frac{8(1+\mathrm{SNR} \cdot \mathrm{MAR})}{\mathrm{SNR} \cdot \mathrm{MAR}} k \log (n-k) \\
\text { From Theorem } 1 \text { (Section IV-C) }\end{array}$ & $\begin{array}{l}\quad m>8 k \log (n-k) \\
\text { From Theorem } 1 \text { (Section IV-C) }\end{array}$ \\
\hline $\begin{array}{c}\text { Necessary and } \\
\text { sufficient for lasso }\end{array}$ & complicated; see [4] & $\begin{array}{c}m>2 k \log (n-k) \\
\text { Wainwright [4] }\end{array}$ \\
\hline $\begin{array}{l}\text { Sufficient for } \\
\text { OMP }\end{array}$ & unknown & $\begin{array}{l}\quad m>2 k \log (n-k) \\
\text { Fletcher and Rangan [29] }\end{array}$ \\
\hline $\begin{array}{c}\text { Sufficient for } \\
\text { thresholding (12) }\end{array}$ & $\begin{array}{c}m>\frac{8(1+\mathrm{SNR})}{\mathrm{MAR} \cdot \mathrm{SNR}} k \log (n-k) \\
\text { Fletcher et al. [5, Thm. 2] }\end{array}$ & $m>\frac{8}{\mathrm{MAR}} k \log (n-k)$ \\
\hline
\end{tabular}

that is within a constant factor of maximum likelihood (ML) detection. This scaling is better than the best known sufficient conditions for lasso and OMP.

The results are not meant to suggest that SequOMP is a good algorithm; other algorithms such as OMP can perform dramatically better. The point is to concretely and provably demonstrate the value of conditional rank information.

\section{B. Related Work}

Under an i.i.d. Gaussian assumption on d, maximum likelihood estimation of $\mathbf{x}$ under a sparsity constraint is equivalent to finding sparse $\hat{\mathbf{x}}$ such that $\|\mathbf{y}-\mathbf{A} \hat{\mathbf{x}}\|_{2}$ is minimized. This is called optimal sparse approximation of $\mathbf{y}$ using dictionary $\mathbf{A}$, and it is NP-hard [6]. Several greedy heuristics (matching pursuit [7] and its variants with orthogonalization [8]-[10] and iterative refinement [11], [12]) and convex relaxations (basis pursuit [13], lasso [14], Dantzig selector [15], and others) have been developed for sparse approximation, and under certain conditions on $\mathbf{A}$ and $\mathbf{y}$ they give optimal or near-optimal performance [16]-[18]. Results showing that near-optimal estimation of $\mathbf{x}$ is obtained with convex relaxations, pointwise over compressible $\mathbf{x}$ and with high probability over some random ensemble for $\mathbf{A}$, form the heart of the compressed sensing literature [19]-[21]. Under a probabilistic model for $\mathbf{x}$ and certain additional assumptions, exact asymptotic performances of several estimators are known [22].

Our interest is in recovery or detection of the support (or sparsity pattern) of $\mathbf{x}$ rather than the estimation of $\mathbf{x}$. In the noiseless case of $\mathbf{d}=0$, optimal estimation of $\mathbf{x}$ can yield $\hat{\mathbf{x}}=\mathbf{x}$ under certain conditions on $\mathbf{A}$; estimation and detection then coincide, and some papers cited above and notably [23] contain relevant results. In the general noisy case, direct analysis of the detection problem has yielded much sharper results.

A standard formulation is to treat $\mathbf{s}$ as a nonrandom parameter vector and $\mathbf{b}$ as either nonrandom with weight $k$ or random with a uniform distribution over the weight- $k$ vectors. The minimum probability of detection error is then attained with ML detection. Sufficient conditions for the success of ML detection are due to Wainwright [3]; necessary conditions based on channel capacity were given by several authors [24]-[27], and conditions more stringent in many regimes and a comparison of results appear in [5]. Necessary and sufficient conditions for lasso were determined by Wainwright [4]. Sufficient conditions for orthogonal matching pursuit (OMP) were given by Tropp and Gilbert [28] and improved by Fletcher and Rangan [29]. Even simpler than OMP is a thresholding algorithm analyzed in a noiseless setting in [30] and with noise in [5]. These results are summarized in Table I, using terminology defined formally in Section II. While thresholded backprojection is unsophisticated from a signal processing point of view, it is simple and commonly used in a variety of fields. Improvements relative to this are needed to justify the use of methods with higher complexity.

Some of our results depend on knowledge of the ordering of conditional powers of entries of $\mathbf{x}$. Several earlier works have introduced other models of partial information about signal support or varying likelihoods of indexes appearing in the support [31]-[33]. Statistical dependencies between components of $\mathbf{x}$ can be exploited very efficiently using a recent extension [34] of the generalized approximate message passing framework [35].

\section{Paper Organization}

The remainder of the paper is organized as follows. The setting is formalized in Section II. In particular, we define all the key problem parameters. Common algorithms and previous results on their performances are then presented in Section III. We will see that there is a potentially-large performance gap between the simplest thresholding algorithm and the optimal ML detection, depending on the signal-to-noise ratio (SNR) and the dynamic range of $\mathbf{x}$. Section IV presents a new detection algorithm, sequential orthogonal matching pursuit (SequOMP), 
that exploits knowledge of conditional ranks. Numerical experiments are reported in Section V. Conclusions are given in Section VI, and proofs are relegated to the Appendix.

\section{PROBLEM FORMULATION}

In the observation model $\mathbf{y}=\mathbf{A x}+\mathbf{d}$, let $\mathbf{A} \in \mathbb{R}^{m \times n}$ and $\mathbf{d} \in \mathbb{R}^{m}$ have i.i.d. $\mathcal{N}(0,1 / m)$ entries. This is a normalization under which the ratio of conditional total signal energy to total noise energy

$$
\operatorname{SNR}(\mathbf{x})=\frac{\mathbf{E}\left[\|\mathbf{A} \mathbf{x}\|^{2} \mid \mathbf{x}\right]}{\mathbf{E}\left[\|\mathbf{d}\|^{2}\right]}
$$

simplifies to

$$
\operatorname{SNR}(\mathbf{x})=\|\mathbf{x}\|^{2} .
$$

This is a random variable because $\mathbf{x}$ is a random vector.

Let $I_{\text {true }}=\left\{j \in\{1,2, \ldots, n\}: x_{j} \neq 0\right\}$ denote the support of $\mathbf{x}$. Using signal model (2), $I_{\text {true }}=\left\{j \in\{1,2, \ldots, n\}: b_{j}=\right.$ $1\}$. The sparsity level of $\mathbf{x}$ is $k=\left|I_{\text {true }}\right|$.

An estimator produces an estimate $\hat{I}=\hat{I}(\mathbf{y})$ of $I_{\text {true }}$ based on the observed noisy vector $\mathbf{y}$. Given an estimator, its probability of $\operatorname{error}^{1} p_{\text {err }}=\operatorname{Pr}\left(\hat{I} \neq I_{\text {true }}\right)$ is taken with respect to randomness in $\mathbf{A}$, noise vector $\mathbf{d}$, and signal $\mathbf{x}$. Our interest is in relating the scaling of problem parameters with the success of various algorithms. For this, we define the following criterion.

Definition 1: Suppose that we are given deterministic sequences $m=m(n), \lambda=\lambda(n)$, and $\mathbf{s}=\mathbf{s}(n) \in \mathbb{R}^{n}$ that vary with $n$. For a given detection algorithm $\hat{I}=\hat{I}(\mathbf{y})$, the probability of error $p_{\text {err }}$ is some function of $n$. We say that the detection algorithm achieves asymptotic reliable detection when $\lim _{n \rightarrow \infty} p_{\text {err }}(n)=0$.

We will see that two key factors influence the ability to detect $I_{\text {true }}$. The first is the total SNR defined above. The second is what we call the minimum-to-average ratio

$$
\operatorname{MAR}(\mathbf{x})=\frac{\min _{j \in I_{\text {true }}}\left|x_{j}\right|^{2}}{\|\mathbf{x}\|^{2} / k}
$$

Like $\operatorname{SNR}(\mathbf{x})$, this is a random variable. Since $I_{\text {true }}$ has $k$ elements, $\|\mathbf{x}\|^{2} / k$ is the average of $\left\{\left|x_{j}\right|^{2}: j \in I_{\text {true }}\right\}$. Therefore, $\operatorname{MAR}(\mathbf{x}) \in(0,1]$ with the upper limit occurring when all the nonzero entries of $\mathbf{x}$ have the same magnitude.

Finally, we define the minimum component $S N R$ to be

$$
\mathrm{SNR}_{\min }(\mathbf{x})=\frac{\min _{j \in I_{\text {true }}} \mathbf{E}\left[\left\|\mathbf{a}_{j} x_{j}\right\|^{2} \mid \mathbf{x}\right]}{\mathbf{E}\left[\|\mathbf{d}\|^{2}\right]}=\min _{j \in I_{\text {true }}}\left|x_{j}\right|^{2},
$$

where $\mathbf{a}_{j}$ is the $j$ th column of $\mathbf{A}$ and the second equality follows from the normalization of chosen for $\mathbf{A}$ and $\mathbf{d}$. The random variable $\mathrm{SNR}_{\text {min }}(\mathbf{x})$ has a natural interpretation: The numerator is the signal power due to the smallest nonzero component in $\mathbf{x}$, while the denominator is the total noise power. The ratio $S N R_{\min }(\mathbf{x})$ thus represents the contribution to the SNR from the smallest nonzero component of $\mathbf{x}$. Observe that (5) and (6) show

$$
\operatorname{SNR}_{\text {min }}(\mathbf{x})=\min _{j \in I_{\text {true }}}\left|x_{j}\right|^{2}=\frac{1}{k} \operatorname{SNR}(\mathbf{x}) \cdot \operatorname{MAR}(\mathbf{x}) .
$$

${ }^{1}$ An alternative to this definition of $p_{\text {err }}$ could be to allow a nonzero fraction of detection errors [26], [27].
We will be interested in estimators that exploit minimal prior knowledge on $\mathbf{x}$ : either only knowledge of sparsity level (through $k$ or $\lambda$ ) or also knowledge of the conditional ranks (through the imposition of (3)). In particular, full knowledge of $\mathbf{s}$ would change the problem considerably because the finite number of possibilities for $\mathbf{x}$ could be exploited.

\section{Common Detection Methods}

In this section, we review several asymptotic analyses for detection of sparse signal support. These previous results hold pointwise over sequences of problems of increasing dimension $n$, i.e., treating $\mathbf{x}$ as an unknown deterministic quantity. That makes these results stronger than results that are limited to the model (2) where the $b_{j}$ s are i.i.d. Bernoulli variables. To reflect the pointwise validity of these results, they are stated in terms of deterministic sequences $\mathbf{x}, m, k, \mathrm{SNR}, \mathrm{MAR}$, and $\mathrm{SNR}_{\text {min }}$ that depend on dimension $n$ and are arbitrary aside from satisfying $m \rightarrow \infty$ and the definitions of the previous section. To simplify the notation, we drop the dependence of $\mathbf{x}, m$ and $k$ on $n$, and SNR, MAR and $\mathrm{SNR}_{\min }$ on $\mathbf{x}(n)$. When the results are tabulated for comparison with each other and with the results of Section IV, we replace $k$ with $\lambda n$; this specializes the results to the model (2).

\section{A. Optimal Detection With No Noise}

To understand the limits of detection, it is useful to first consider the minimum number of measurements when there is no noise. Suppose that $k$ is known to the detector. With no noise, the observed vector is $\mathbf{y}=\mathbf{A x}$, which will belong to one of $J=\left(\begin{array}{l}n \\ k\end{array}\right)$ subspaces spanned by $k$ columns of A. If $m>k$, then these subspaces will be distinct with probability 1 . Thus, an exhaustive search through the subspaces will reveal which subspace y belongs to and thus determine the support $I_{\text {true }}$. This shows that with no noise and no computational limits, the scaling in measurements of

$$
m>k
$$

is sufficient for asymptotic reliable detection.

Conversely, if no prior information is known at the detector other than $\mathbf{x}$ being $k$-sparse, then the condition (9) is also necessary. If $m \leq k$, then for almost all $\mathbf{A}$, any $k$ columns of $\mathbf{A}$ span $\mathbb{R}^{m}$. Consequently, any observed vector $\mathbf{y}=\mathbf{A x}$ is consistent with any support of weight $k$. Thus, the support cannot be determined without further prior information on the signal $\mathbf{x}$.

Note that we are considering correct detection with probability 1 (over the random choice of $\mathbf{A}$ ) for a single $k$-sparse $\mathbf{x}$. It is elementary to show that correct detection with probability 1 (again over the random choice of $\mathbf{A}$ ) for all $k$-sparse $\mathbf{x}$ requires $m \geq 2 k$.

\section{B. ML Detection With Noise}

Now suppose there is noise. Since $\mathbf{x}$ is an unknown deterministic quantity, the probability of error in detecting the support is minimized by maximum likelihood (ML) detection. Since the noise $\mathbf{d}$ is Gaussian, the ML detector finds the $k$-dimensional subspace spanned by $k$ columns of $\mathbf{A}$ containing the maximum energy of $\mathbf{y}$. 
The ML estimator was first analyzed by Wainwright [3]. He shows that there exists a constant $C>0$ such that if

$$
\begin{aligned}
m & \geq C \max \left\{\frac{1}{\mathrm{MAR} \cdot \mathrm{SNR}} k \log (n-k), k \log (n / k)\right\} \\
& =C \max \left\{\frac{1}{\mathrm{SNR}_{\text {min }}} \log (n-k), k \log (n / k)\right\}
\end{aligned}
$$

then ML will asymptotically detect the correct support. The equivalence of the two expressions in (10) is due to (8). Also, [5, Thm. 1] (generalized in [36, Thm. 1]) shows that, for any $\delta>0$, the condition

$$
\begin{aligned}
m & \geq \frac{2(1-\delta)}{\mathrm{MAR} \cdot \mathrm{SNR}} k \log (n-k)+k \\
& =\frac{2(1-\delta)}{\mathrm{SNR}_{\min }} \log (n-k)+k,
\end{aligned}
$$

is necessary. Observe that when SNR $\cdot$ MAR $\rightarrow \infty$, the lower bound (11) approaches $m \geq k$, matching the noise-free case (9) as expected.

These necessary and sufficient conditions for ML appear in Table I with smaller terms and the infinitesimal $\delta$ omitted for simplicity.

\section{Thresholding}

The simplest method to detect the support is to use a thresholding rule of the form

$$
\hat{I}_{\mathrm{T}}=\{j \in\{1,2, \ldots, n\}: \rho(j)>\mu\},
$$

where $\mu>0$ is a threshold parameter and $\rho(j)$ is the correlation coefficient:

$$
\rho(j)=\frac{\left|\mathbf{a}_{j}^{\prime} \mathbf{y}\right|^{2}}{\left\|\mathbf{a}_{j}\right\|^{2}\|\mathbf{y}\|^{2}}, \quad j=1,2, \ldots, n .
$$

Thresholding has been analyzed in [5], [30], [37]. In particular, [5, Thm. 2] is the following: Suppose

$$
\begin{aligned}
m & >\frac{2(1+\delta)(1+\mathrm{SNR}) k L(k, n)}{\mathrm{SNR} \cdot \mathrm{MAR}} \\
& =\frac{2(1+\delta)(1+\mathrm{SNR}) L(k, n)}{\mathrm{SNR}_{\min }}
\end{aligned}
$$

where $\delta>0$ and

$$
L(k, n)=[\sqrt{\log (n-k)}+\sqrt{\log (k)}]^{2} .
$$

Then there exists a sequence of detection thresholds $\mu=\mu(n)$ such that $\hat{I}_{\mathrm{T}}$ achieves asymptotic reliable detection of the support. As before, the equivalence of the two expressions in (13) is due to (8).

Comparing the sufficient condition (13) for thresholding with the necessary condition (11), we see two distinct problems with thresholding:

- Constant offset: The scaling (13) for thresholding shows a factor $L(k, n)$ instead of $\log (n-k)$ in (11). It is easily verified that, for $k / n \in(0,1 / 2)$,

$$
\log (n-k)<L(k, n)<4 \log (n-k),
$$

so this difference in factors alone could require that thresholding use up to 4 times more measurements than ML for asymptotic reliable detection.

Combining the inequality (15) with (13), we see that the more stringent, but simpler, condition

$$
m>\frac{8(1+\delta)(1+\mathrm{SNR})}{\mathrm{SNR} \cdot \mathrm{MAR}} k \log (n-k)
$$

is also sufficient for asymptotic reliable detection with thresholding. This simpler condition is shown in Table I, where we have omitted the infinitesimal $\delta$ quantity to simplify the table entry.

- SNR saturation: In addition to the $L(k, n) / \log (n-k)$ offset, thresholding also requires a factor of $1+$ SNR more measurements than ML. This $1+$ SNR factor has a natural interpretation as intrinsic interference: When detecting any one component of the vector $\mathbf{x}$, thresholding sees the energy from the other $n-1$ components of the signal as interference. This interference is distinct from the additive noise $\mathbf{d}$, and it increases the effective noise by a factor of $1+$ SNR.

The intrinsic interference results in a large performance gap at high SNRs. In particular, as SNR $\rightarrow \infty,(13)$ reduces to

$$
m>\frac{2(1+\delta) k L(k, n)}{\operatorname{MAR}} .
$$

In contrast, ML may be able to succeed with a scaling $m=$ $O(k)$ for high SNRs.

\section{Lasso and OMP Detection}

While ML has clear advantages over thresholding, it is not computationally tractable for large problems. One practical method is lasso [14], also called basis pursuit denoising [13]. The lasso estimate of $\mathbf{x}$ is obtained by solving the convex optimization

$$
\hat{\mathbf{x}}=\underset{\mathbf{x}}{\arg \min }\left(\|\mathbf{y}-\mathbf{A} \mathbf{x}\|_{2}^{2}+\mu\|\mathbf{x}\|_{1}\right),
$$

where $\mu>0$ is an algorithm parameter that encourages sparsity in the solution $\hat{\mathbf{x}}$. The nonzero components of $\hat{\mathbf{x}}$ can then be used as an estimate of $I_{\text {true }}$.

Wainwright [4] has given necessary and sufficient conditions for asymptotic reliable detection with lasso. Partly because of freedom in the choice of a sequence of parameters $\mu(n)$, the finite SNR results are difficult to interpret. Under certain conditions with SNR growing unboundedly with $n$, matching necessary and sufficient conditions can be found. Specifically, if $m$, $n$ and $k \rightarrow \infty$, with SNR $\cdot$ MAR $\rightarrow \infty$, the scaling

$$
m>2 k \log (n-k)+k+1
$$

is both necessary and sufficient for asymptotic reliable detection.

Another common approach to support detection is the OMP algorithm [8]-[10]. This was analyzed by Tropp and Gilbert [28] in a setting with no noise. This was generalized to the present setting with noise by Fletcher and Rangan [29]. The result is very similar to condition (18): If $m, n$ and $k \rightarrow \infty$, with 
SNR $\cdot$ MAR $\rightarrow \infty$, a sufficient condition for asymptotic reliable recovery is

$$
m>2 k \log (n-k) .
$$

The main result of [29] also allows uncertainty in $k$.

The conditions (18) and (19) are both shown in Table I. As usual, the table entries are simplified by including only the leading terms.

The lasso and OMP scaling laws, (18) and (19), can be compared with the high SNR limit for the thresholding scaling law in (17). This comparison shows the following:

- Removal of the constant offset: The $L(k, n)$ factor in the thresholding expression is replaced by a $\log (n-k)$ factor in the lasso and OMP scaling laws. Similar to the discussion above, this implies that lasso and OMP could require up to 4 times fewer measurements than thresholding.

- Dynamic range: In addition, both the lasso and OMP methods do not have a dependence on MAR. This gain can be large when there is high dynamic range, i.e., MAR is near zero.

- Limits at high SNR: We also see from (18) and (19) that both lasso and OMP are unable to achieve the scaling $m=O(k)$ that may be achievable with ML at high SNR. Instead, both lasso and OMP have the scaling $m=O(k \log (n-k))$, similar to the minimum scaling possible with thresholding.

\section{E. Other Sparsity Detection Algorithms}

Recent interest in compressed sensing has led to a plethora of algorithms beyond OMP and lasso. Empirical evidence suggests that the most promising algorithms for support detection are the sparse Bayesian learning methods developed in the machine learning community [38] and introduced into signal processing applications in [39], with related work in [40]. Unfortunately, a comprehensive summary of these algorithms is far beyond the scope of this paper. Our interest is not in finding the optimal algorithm, but rather to explain qualitative differences between algorithms and to demonstrate the value of knowing conditional ranks a priori.

\section{Sequential Orthogonal Matching Pursuit}

The results summarized in the previous section suggest a large performance gap between ML detection and practical algorithms such as thresholding, lasso and OMP, especially when the SNR is high. Specifically, as the SNR increases, the performance of these practical methods saturates at a scaling in the number of measurements that can be significantly higher than that for ML.

In this section, we introduce an OMP-like algorithm, which we call sequential orthogonal matching pursuit, that under favorable conditions can break this barrier. Specifically, in some cases, the performance of SequOMP does not saturate at high SNR.

\section{A. Algorithm: SequOMP}

Given a received vector $\mathbf{y}$, threshold level $\mu>0$, and detection order $\pi$ (a permutation on $\{1,2, \ldots, n\}$ ), the algorithm produces an estimate $\hat{I}_{\mathrm{S}}$ of the support $I_{\text {true }}$ with the following steps:
1) Initialize the counter $j=1$ and set the initial support estimate to empty: $\hat{I}(0)=\emptyset$.

2) Compute $\mathbf{P}(j) \mathbf{a}_{\pi(j)}$ where $\mathbf{P}(j)$ is the projection operator onto the orthogonal complement of the span of $\left\{\mathbf{a}_{\pi(\ell)}, \pi(\ell) \in \hat{I}(j-1)\right\}$.

3) Compute the squared correlation between $\mathbf{P}(j) \mathbf{a}_{\pi(j)}$ and $\mathbf{P}(j) \mathbf{y}$ :

$$
\rho(j)=\frac{\left|\mathbf{a}_{\pi(j)}^{\prime} \mathbf{P}(j) \mathbf{y}\right|^{2}}{\left\|\mathbf{P}(j) \mathbf{a}_{\pi(j)}\right\|^{2}\|\mathbf{P}(j) \mathbf{y}\|^{2}} .
$$

4) If $\rho(j)>\mu$, add the index $\pi(j)$ to $\hat{I}(j-1)$. That is, $\hat{I}(j)=\hat{I}(j-1) \cup\{j\}$. Otherwise, set $\hat{I}(j)=\hat{I}(j-1)$.

5) Increment $j$ to $j+1$. If $j \leq n$ return to step 2 .

6) The final estimate of the support is $\hat{I}_{\mathrm{S}}=\hat{I}(n)$.

The SequOMP algorithm can be thought of as an iterative version of thresholding with the difference that, after a nonzero component is detected, subsequent correlations are performed only in the orthogonal complement to the corresponding column of $\mathbf{A}$. The method is identical to the standard OMP algorithm of [8]-[10], except that SequOMP passes through the data only once, in a fixed order. For this reason, SequOMP is computationally simpler than standard OMP.

As simulations will illustrate later, SequOMP generally has much worse performance than standard OMP. It is not intended as a competitive practical alternative. Our interest in the algorithm lies in the fact that we can prove positive results for SequOMP. Specifically, we will be able to show that this simple algorithm, when used in conjunction with known conditional ranks, can achieve a fundamentally better scaling at high SNRs than what has been proven is achievable with methods such as lasso and OMP.

\section{B. Sequential OMP Performance}

The analyses in Section III hold for deterministic vectors $\mathbf{x}$. Recall the partially-random signal model (2) where $b_{j}$ is a $\operatorname{Bernoulli}(\lambda)$ random variable while the value of $x_{j}$ conditional on $x_{j}$ being nonzero remains deterministic; i.e., $s_{j}$ is deterministic.

Let $p_{j}$ denote the conditional energy of $x_{j}$, conditioned on $b_{j}=1$ (i.e., $j \in I_{\text {true }}$ ). Then

$$
p_{j}=s_{j}^{2}, \quad j=1,2, \ldots, n .
$$

We will call $\left\{p_{j}\right\}_{j=1}^{n}$ the power profile. Since $\operatorname{Pr}\left(b_{j}=1\right)=\lambda$ for every $j$, the average value of $\operatorname{SNR}(\mathbf{x})$ in (4) is given by

$$
\mathrm{SNR}=\mathbf{E}[\operatorname{SNR}(\mathbf{x})]=\lambda \sum_{j=1}^{n} p_{j} .
$$

Also, in analogy with MAR(x) and $\mathrm{SNR}_{\min }(\mathbf{x})$ in (6) and (7), define

$$
\begin{aligned}
\mathrm{SNR}_{\min } & =\min _{j} p_{j}, \\
\mathrm{MAR} & =\frac{\lambda n}{\mathrm{SNR}} \min _{j} p_{j}=\frac{\lambda n \mathrm{SNR}_{\min }}{\mathrm{SNR}} .
\end{aligned}
$$

Note that the power profile $p_{j}$ and the quantities $\mathrm{SNR}, \mathrm{SNR}$ min and MAR as defined above are deterministic.

To simplify notation, we henceforth assume $\pi$ is the identity permutation, i.e., the detection order in SequOMP is simply $(1,2, \ldots, n)$. A key parameter in analyzing the performance of 
SequOMP is what we will call the minimum signal-to-interference and noise ratio (MSINR)

$$
\gamma=\min _{\ell=1, \ldots, n} p_{\ell} / \hat{\sigma}^{2}(\ell)
$$

where $\hat{\sigma}^{2}(\ell)$ is given by

$$
\hat{\sigma}^{2}(\ell)=1+\lambda \sum_{j=\ell+1}^{n} p_{j}, \quad \ell=1,2, \ldots, n .
$$

The parameters $\gamma$ and $\hat{\sigma}^{2}(\ell)$ have simple interpretations: Suppose SequOMP has correctly detected $b_{j}$ for all $j<\ell$. Then, in detecting $b_{\ell}$, the algorithm sees the noise $\mathbf{d}$ with power $\mathbf{E}\left[\|\mathbf{d}\|^{2}\right]=1$ plus, for each component $j>\ell$, an interference power $p_{j}$ with probability $\lambda$. Hence, $\hat{\sigma}^{2}(\ell)$ is the total average interference power seen when detecting $b_{\ell}$, assuming perfect cancellation up to that point. Since the conditional power of $x_{\ell}$ is $p_{\ell}$, the ratio $p_{\ell} / \hat{\sigma}^{2}(\ell)$ in (22a) represents the average SINR seen while detecting component $\ell$. The value $\gamma$ is the minimum SINR over all $n$ components.

Theorem 1: Let $\lambda=\lambda(n), m=m(n)$, and the power profile $\left\{p_{j}\right\}_{j=1}^{n}=\left\{p_{j}(n)\right\}_{j=1}^{n}$ be deterministic quantities varying with $n$ that satisfy

$$
\begin{aligned}
\lim _{n \rightarrow \infty} \lambda n & =\infty, \quad \lim _{n \rightarrow \infty}(1-\lambda) n=\infty, \\
\lim _{n \rightarrow \infty} \frac{m-\lambda n}{\log (\lambda n)} & =\infty, \quad \lim _{n \rightarrow \infty} \frac{m-\lambda n}{\log ((1-\lambda) n)}=\infty .
\end{aligned}
$$

Also, assume the sequence of power profiles satisfies the limit

$$
\lim _{n \rightarrow \infty} \max _{i=1, \ldots, n-1} \log (n) \hat{\sigma}^{-4}(i) \sum_{j>i}^{n} p_{j}^{2}=0 .
$$

Finally, assume that for all $n$,

$$
m \geq \frac{2(1+\delta)(1+\sqrt{\gamma})^{2} L(\lambda n, n)}{\gamma}+\lambda n,
$$

for some $\delta>0$ where $L(\cdot, \cdot)$ is defined in (14) and $\gamma$ is defined in (22a). Then, there exists a sequence of thresholds, $\mu=\mu(n)$, such that SequOMP with detection order $(1,2, \ldots, n)$ will achieve asymptotic reliable detection. The sequence of threshold levels can be selected independent of the sequence of power profiles.

Proof: See Appendix A.

The theorem provides a simple sufficient condition on the number of measurements as a function of the MSINR $\gamma$, probability $\lambda$, and dimension $n$. The condition (23c) is somewhat technical; we will verify its validity in examples. The remainder of this section discusses some of the implications of this theorem.

\section{Most Favorable Detection Order With Known Conditional Ranks}

Suppose that the ordering of the conditional power levels $\left\{p_{j}\right\}_{j=1}^{n}$ is known at the detector, but possibly not the values themselves. Reordering the power profile is equivalent to changing the detection order, so we seek the most favorable ordering of the power profile. Since $\hat{\sigma}^{2}(\ell)$ defined in $(22 b)$ involves the sum of the tail of the power profile, the MSINR defined in (22a) is maximized when the power profile is non-increasing:

$$
p_{1} \geq p_{2} \geq \cdots \geq p_{n}=\mathrm{SNR}_{\min } .
$$

In other words, the best detection order for SequOMP is from strongest component to weakest component.

Using (25), it can be verified that the MSINR $\gamma$ is bounded below by

$$
\gamma \geq \frac{\mathrm{SNR}_{\text {min }}}{1+\lambda n \mathrm{SNR}_{\text {min }}}=\frac{\mathrm{SNR} \cdot \mathrm{MAR}}{\lambda n(1+\mathrm{SNR} \cdot \mathrm{MAR})}
$$

Furthermore, in cases of interest $\gamma \rightarrow 0$, so the sufficiency of the scaling (24) shows that

$$
m \geq \frac{2(1+\delta) \lambda n(1+\mathrm{SNR} \cdot \mathrm{MAR})}{\mathrm{SNR} \cdot \mathrm{MAR}} L(\lambda n, n)+\lambda n
$$

is sufficient for asymptotic reliable detection. This expression is shown in Table I with the additional simplification that $L(\lambda n, n) \leq 4 \log (n(1-\lambda))$ for $\lambda \in(0,1 / 2)$. To keep the notation consistent with the expressions for the other entries in the table, we have used $k$ for $\lambda n$, which is the average number of nonzero entries of $\mathbf{x}$.

When SNR $\rightarrow \infty$, (27) simplifies to

$$
m \geq 2(1+\delta) \lambda n L(\lambda n, n)+\lambda n .
$$

This is identical to the lasso and OMP performance except for the factor $L(\lambda n, n) / \log ((1-\lambda) n)$, which lies in $(1,4)$ for $\lambda \in$ $(0,1 / 2)$. In particular, the minimum number of measurements does not depend on MAR; therefore, similar to lasso and OMP, SequOMP can theoretically detect components that are much below the average power at high SNRs. More generally, we can say that knowledge of the conditional ranks of the powers enables a very simple algorithm to achieve resistance to large dynamic ranges.

\section{Optimal Power Shaping}

The MSINR lower bound in (26) is achieved as $n \rightarrow \infty$ and the power profile is constant (all $p_{j}$ 's are equal). Thus, opposite to thresholding, a constant power profile is in some sense the worst power profile for a given $\mathrm{SNR}_{\min }$ for the SequOMP algorithm.

This raises the question: What is the most favorable power profile? Any power profile maximizing the MSINR $\gamma$ subject to a constraint on total SNR (21) will achieve the minimum in (22a) for every $\ell$ and thus satisfy

$$
p_{\ell}=\gamma\left(1+\lambda \sum_{j=\ell+1}^{n} p_{j}\right), \quad \ell=1,2, \ldots, n .
$$

The solution to (29) and (21) is given by

$$
p_{\ell}=\gamma_{\mathrm{opt}}\left(1+\gamma_{\mathrm{opt}} \lambda\right)^{n-\ell}, \quad \ell=1,2, \ldots, n,
$$

where

$$
\gamma_{\mathrm{opt}}=\frac{1}{\lambda}\left[(1+\mathrm{SNR})^{1 / n}-1\right] \approx \frac{1}{\lambda n} \log (1+\mathrm{SNR})
$$


and the approximation holds for large $n .{ }^{2}$ Again, some algebra shows that when $\lambda$ is bounded away from zero, the power profile in (30) will satisfy the technical condition (23c) when $\log (1+$ $\mathrm{SNR})=o(n / \log (n))$.

The power profile (30a) is exponentially decreasing in the index order $\ell$. Thus, components early in the detection sequence are allocated exponentially higher power than components later in the sequence. This allocation insures that early components have sufficient power to overcome the interference from all the components later in the detection sequence that are not yet cancelled.

Substituting (30b) into (24), we see that the scaling

$$
m \geq \frac{2(1+\delta) L(\lambda n, n)}{\log (1+\mathrm{SNR})} \lambda n+\lambda n
$$

is sufficient for SequOMP to achieve asymptotic reliable detection with the best-case power profile. This expression is shown in Table I, again with the additional simplification that $L(\lambda n, n) \leq 4 \log (n(1-\lambda))$ for $\lambda \in(0,1 / 2)$.

\section{E. SNR Saturation}

As discussed earlier, a major problem with thresholding, lasso, and OMP is that their performances "saturate" with high SNR. That is, even as the SNR scales to infinity, the minimum number of measurements scales as $m=\Theta(\lambda n \log ((1-\lambda) n)$. In contrast, optimal ML detection can achieve a scaling $m=O(\lambda n)$, when the SNR is sufficiently high.

A consequence of (31) is that SequOMP with exponential power shaping can overcome this barrier. Specifically, if we take the scaling of SNR $=\Theta(\lambda n)$ in (31), apply the bound $L(\lambda n, n) \leq 4 \log (n(1-\lambda))$ for $\lambda \in(0,1 / 2)$, and assume that $\lambda$ is bounded away from zero, we see that asymptotically, SequOMP requires only $m \geq 9 \lambda n$ measurements. In this way, unlike thresholding and lasso, SequOMP is able to succeed with scaling $m=O(\lambda n)$ when SNR $\rightarrow \infty$. In fact, if SNR grows slightly faster so that it satisfies $L(\lambda n, n)=o(\log (1+\mathrm{SNR}))$ while still satisfying $\log (1+\mathrm{SNR})=o(n / \log (n))$, then (31) leads to an asymptotic sufficient condition of $m>n$.

\section{F. Power Shaping With Sparse Bayesian Learning}

The fact that power shaping can provide benefits when combined with certain iterative detection algorithms confirms the observations in the work of Wipf and Rao [41]. That work considers signal detection with a certain sparse Bayesian learning (SBL) algorithm. They show the following result: Suppose $\mathbf{x}$ has $k$ nonzero components and $p_{i}, i=1,2, \ldots, k$, is the power of the $i$ th largest component. Then, for a given measurement matrix $\mathbf{A}$, there exist constants $\nu_{i}>1$ such that if

$$
p_{i} \geq \nu_{i} p_{i-1}, \quad i=2,3, \ldots, k,
$$

the SBL algorithm will correctly detect the sparsity pattern of $\mathbf{x}$.

${ }^{2}$ The solution (30) is the $\theta=0$ case of a more general result in Section IV.G; see (35).
The condition (32) shows that a certain growth in the powers can guarantee correct detection. The parameters $\nu_{i}$ however depend in some complex manner on the matrix $\mathbf{A}$, so the appropriate growth is difficult to compute. They also provide strong empirical evidence that shaping the power with certain profiles can greatly reduce the number of measurements needed.

The results in this paper add to Wipf and Rao's observations showing that growth in the powers can also assist SequOMP. Moreover, for SequOMP, we can explicitly derive the optimal power profile for certain large random matrices.

This is not to say that SequOMP is better than SBL. In fact, empirical results in [39] suggest that SBL will outperform OMP, which will in turn do better than SequOMP. As we have stressed before, the point of analyzing SequOMP here is that we can derive concrete analytic results. These results may provide guidance for more sophisticated algorithms.

\section{G. Robust Power Shaping}

The above analysis shows certain benefits of SequOMP used in conjunction with power shaping. The results are proven for reliable detection of all entries of the support in a limit of unbounded block length (see Definition 1). In problems of finite size or at operating points where a nonzero fraction of errors is tolerable, the power shaping above may hurt performance.

When a nonzero component is not detected in SequOMP, that component's energy is not cancelled out and remains as interference for all subsequent components in the detection sequence. With power shaping, components early in the detection sequence have much higher power than components later in the sequence. Compared to a case with the same SNR and a constant power profile, the use of power shaping reduces the probability of an early missed detection but increases the harm in subsequent steps that comes from such a missed detection. As block length increases, the probability of missed detection can be driven to zero. But at any finite block length, the probability of a missed detection early in the sequence will always be nonzero.

The work [42] observed a similar problem when successive interference cancellation is used in a CDMA uplink. To mitigate the problem, [42] proposed to adjust the power allocations to make them more robust to detection errors early in the detection sequence. The same technique, which we will call robust power shaping, can be applied to SequOMP as follows.

The condition (29) is motivated by maintaining a constant MSINR through the detection process, assuming all components with indexes $j<\ell$ have been correctly detected and subtracted. An alternative, following [42], is to assume that some fixed fraction $\theta \in[0,1]$ of the energy of components early in the detection sequence is not cancelled out due to missed detections. We will call $\theta$ the leakage fraction. With nonzero leakage, the condition (29) is replaced by

$$
p_{\ell}=\gamma\left(1+\theta \lambda \sum_{j=1}^{\ell-1} p_{j}+\lambda \sum_{j=\ell+1}^{n} p_{j}\right), \quad \ell=1,2, \ldots, n
$$

For given $\gamma, \lambda$, and $\theta,(33)$ in a system of linear equations that determines the power profile $\left\{p_{\ell}\right\}_{\ell=1}^{n}$; one can vary $\gamma$ until the power profile provides the desired SNR according to (21). 

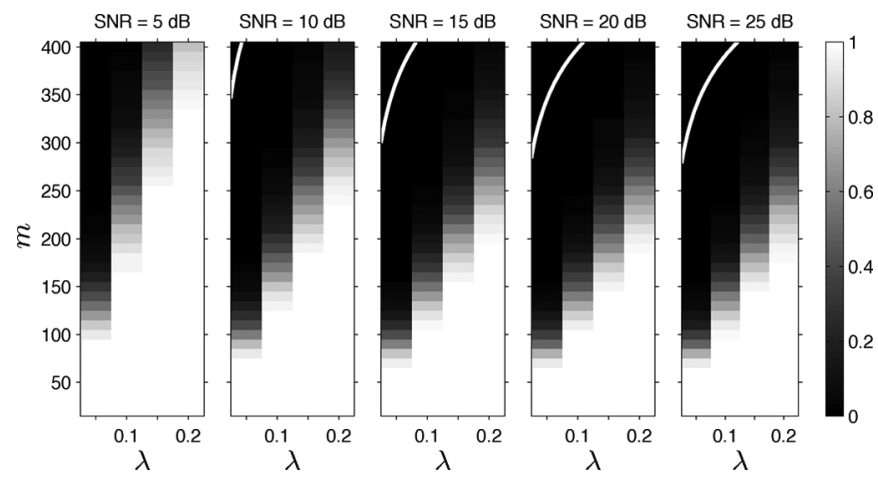

Fig. 1. Probability of error in full support recovery with SequOMP for a signal with $n=100$ components when the power profile is optimized as in (35) with leakage fraction $\theta=0.1$. Each shaded box presents the result of 2000 Monte Carlo trials with $\lambda \in\{0.05,0.1,0.15,0.2\}, m \in\{20,40, \ldots, 400\}$ and SNR as indicated. The white line shows the theoretical sufficient condition on $m$ obtained from Theorem 1 .

A closed-form solution to (33) provides some additional insight. Adding and subtracting SNR inside the parentheses in (33) while also using (21) yields

$$
p_{\ell}=\gamma(1+\underbrace{\mathrm{SNR}-\lambda \sum_{j=1}^{n} p_{j}}_{=0}+\theta \lambda \sum_{j=1}^{\ell-1} p_{j}+\lambda \sum_{j=\ell+1}^{n} p_{j}),
$$

which can be rearranged to

$$
(1+\gamma \lambda) p_{\ell}=\gamma\left(1+\mathrm{SNR}-(1-\theta) \lambda \sum_{j=1}^{\ell-1} p_{j}\right) .
$$

Using standard techniques for solving linear constant-coefficient difference equations,

$$
p_{j}=\frac{\mathrm{SNR}}{\lambda} \cdot \frac{(1-\zeta) \zeta^{j-1}}{1-\zeta^{n}}
$$

where

$$
\zeta=\frac{1+\gamma \lambda \theta}{1+\gamma \lambda}
$$

and

$$
\gamma=\frac{1}{\lambda} \cdot \frac{1-\left(\frac{1+\theta \mathrm{SNR}}{1+\mathrm{SNR}}\right)^{1 / n}}{\left(\frac{1+\theta \mathrm{SNR}}{1+\mathrm{SNR}}\right)^{1 / n}-\theta}
$$

Notice that $\theta<1$ implies $\zeta<1$, so the power profile (35a) is decreasing as in the case without leakage in Section IV-D. Setting $\theta=0$ recovers (30).

\section{Numerical Simulations}

\section{A. Basic Validation of Sufficient Condition}

We first compare the actual performance of the SequOMP algorithm with the sufficient condition for support recovery in Theorem 1. Fig. 1 shows the simulated probability of error $p_{\text {err }}$ obtained using SequOMP at various SNR levels, probabilities of

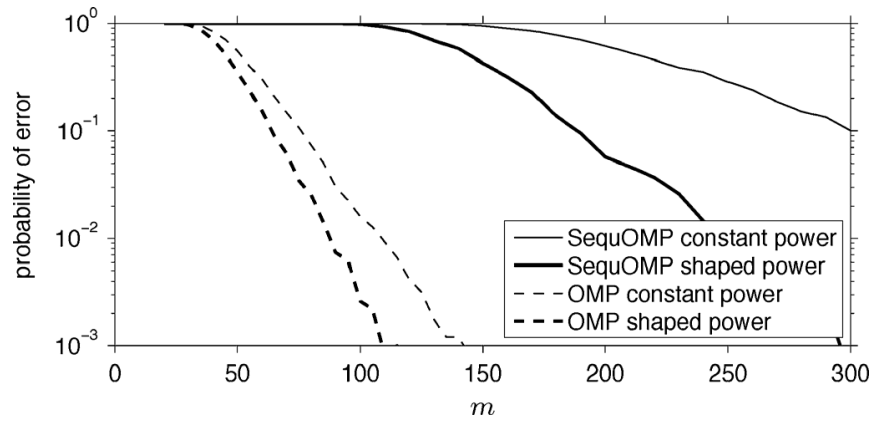

Fig. 2. Comparison of SequOMP and OMP, with and without power shaping Probability of error in full support recovery is plotted as a function of $m$ with number of components $n=100$, probability of an entry being nonzero $\lambda=$ 0.1 , and SNR $=25 \mathrm{~dB}$. The power profile is either constant or optimized as in (35) with leakage fraction $\theta=0.1$.

nonzero components $\lambda$, and numbers of measurements $m$. In all these simulations, the number of components was fixed to $n=$ 100 , and each shaded box represents an empirical probability of error over 2000 independent Monte Carlo trials. The robust power profile of Section IV-G is used with a leakage fraction $\theta=0.1$. Here and in subsequent simulations, the threshold $\mu$ is set to the level specified in (39) in the proof of Theorem 1 in Appendix A. ${ }^{3}$

The white line in Fig. 1 represents the number of measurements $m$ for which Theorem 1 would theoretically guarantee reliable detection of the support at infinite block lengths. To apply the theorem, we used the MSINR $\gamma$ from (35c). At the block lengths considered in these simulations, the probability of error at the theoretical sufficient condition is small, typically under $0.1 \%$. The theoretical sufficient condition shows the same trends as the empirical results.

\section{B. Effect of Power Shaping}

Fig. 2 compares the performances of SequOMP and OMP, with and without power shaping. In the simulations, $n=100$, $\lambda=0.1$, and the total SNR is $25 \mathrm{~dB}$. When power shaping is used, the power profile is determined through (35) with leakage fraction $\theta=0.1$. Otherwise, the power profile is constant. The number of measurements $m$ was varied, and for each $m$, the probability of error was estimated with 5000 independent Monte Carlo trials.

As expected from the theoretical analysis in this paper, with the total SNR kept constant, the performance of SequOMP is improved by optimization of the power profile. As also is to be expected, SequOMP is considerably worse than OMP in terms of error probability for a given number of measurements or number of measurements needed to achieve a given error probability. Our interest in SequOMP is that it is amenable to analysis; OMP presumably performs better than SequOMP in any setting of interest, but it does not do so for every problem instance, so our analysis does not carry over to OMP rigorously.

The simulation in Fig. 2 shows that power shaping provides gains with OMP as well. As discussed in Section IV-F, this is consistent with observations in the work of Wipf and Rao [41].

\footnotetext{
${ }^{3}$ Simulations presented in [43] use a different choice of $\mu$. There, $\mu$ is adjusted to achieve a fixed false alarm probability and all plotted quantities are missed detection probabilities. The conclusions are qualitatively similar.
} 


\section{CONCLUSION}

Methods such as OMP and lasso, which are widely used in sparse signal support detection problems, exhibit advantages over thresholding but still fall far short of the performance of optimal (ML) detection at high SNRs. Analysis of the SequOMP algorithm has shown that knowledge of conditional rank of signal components enables performance similar to OMP and lasso at a lower complexity. Furthermore, in the most favorable situations, conditional rank knowledge changes the fundamental scaling of performance with SNR so that performance no longer saturates with SNR.

\section{APPENDIX}

\section{PROOF OF THEOREM 1}

\section{A. Proof Outline}

At a high level, the proof of Theorem 1 is similar to the proof of [5, Thm. 2], the thresholding condition (16). One of the difficulties in the proof is to handle the dependence between random events at different iterations of the SequOMP algorithm. To avoid this difficulty, we first show an equivalence between the success of SequOMP and an alternative sequence of events that is easier to analyze. After this simplification, small modifications handle the cancellations of detected vectors.

Fix $n$ and define $I_{\text {true }}(j)=\left\{\ell: \ell \in I_{\text {true }}, \ell \leq j\right\}$, which is the set of elements of the true support with indices $\ell \leq j$. Observe that $I_{\text {true }}(0)=\emptyset$ and $I_{\text {true }}(n)=I_{\text {true }}$.

Let $\mathbf{P}_{\text {true }}(j)$ be the projection operator onto the orthogonal complement of $\left\{\mathbf{a}_{\ell}, \ell \in I_{\text {true }}(j-1)\right\}$, and define

$$
\rho_{\text {true }}(j)=\frac{\left|\mathbf{a}_{j}^{\prime} \mathbf{P}_{\text {true }}(j) \mathbf{y}\right|^{2}}{\left\|\mathbf{P}_{\text {true }}(j) \mathbf{a}_{j}\right\|^{2}\left\|\mathbf{P}_{\text {true }}(j) \mathbf{y}\right\|^{2}} .
$$

A simple induction argument shows that SequOMP correctly detects the support if and only if, at each iteration $j$, the variables $\hat{I}(j), \mathbf{P}(j)$ and $\rho(j)$ defined in the algorithm are equal to $I_{\text {true }}(j), \mathbf{P}_{\text {true }}(j)$ and $\rho_{\text {true }}(j)$, respectively. Therefore, if we define $\hat{I}=\left\{j: \rho_{\text {true }}(j)>\mu\right\}$, then SequOMP correctly detects the support if and only if $\hat{I}=I_{\text {true }}$. In particular, $p_{\text {err }}(n)=\operatorname{Pr}\left(\hat{I} \neq I_{\text {true }}\right)$.

To prove that $p_{\text {err }}(n) \rightarrow 0$ it suffices to show that there exists a sequence of threshold levels $\mu(n)$ such that

$$
\begin{aligned}
& \liminf _{n \rightarrow \infty} \min _{j \in I_{\text {true }}(n)} \frac{\rho_{\text {true }}(j)}{\mu}>1, \\
& \limsup _{n \rightarrow \infty} \max _{j \notin I_{\text {true }}(n)} \frac{\rho_{\text {true }}(j)}{\mu}<1,
\end{aligned}
$$

hold in probability. The first limit (37a) ensures that all the components in the true support will not be missed and will be called the zero missed detection condition. The second limit (37b) ensures that all the components not in the true support will not be falsely detected and will be called the zero false alarm condition.

Set the sequence of threshold levels as follows. Since $\delta>0$, we can find an $\epsilon>0$ such that

$$
(1+\delta) \geq(1+\epsilon)^{2}
$$

For each $n$, let the threshold level be

$$
\mu=(1+\epsilon)(1+\sqrt{\gamma})^{2} \frac{2 \log (n(1-\lambda))}{m-\lambda n} .
$$

The asymptotic lack of missed detections and false alarms with these thresholds are proven in Appendices D and E, respectively. In preparation for these sections, Appendix B reviews some facts concerning tail bounds on chi-squared and beta random variables and Appendix $\mathrm{C}$ presents some preliminary computations.

\section{B. Chi-Squared and Beta Random Variables}

The proof requires a number of simple facts concerning chi-squared and beta random variables. These variables are reviewed in [44]. We omit all the proofs in this subsection and instead reference very closely related lemmas in [5].

A random variable $u$ has a chi-squared distribution with $r$ degrees of freedom if it can be written as $u=\sum_{i=1}^{r} z_{i}^{2}$, where $z_{i}$ are i.i.d. $\mathcal{N}(0,1)$.

Lemma 1 ([5], Lemma 2): Suppose $\mathbf{x} \in \mathbb{R}^{r}$ has a Gaussian distribution $\mathcal{N}\left(0, \sigma^{2} I_{r}\right)$. Then:

a) $\|\mathbf{x}\|^{2} / \sigma^{2}$ is chi-squared with $r$ degrees of freedom; and

b) if $\mathbf{y}$ is any other $r$-dimensional random vector that is nonzero with probability one and independent of $\mathbf{x}$, then the variable $u=\left|\mathbf{x}^{\prime} \mathbf{y}\right|^{2}\left(\sigma^{2}\|\mathbf{y}\|^{2}\right)$ is a chi-squared random variable with one degree of freedom.

The following two lemmas provide standard tail bounds.

Lemma 2 (Similar to [5], Lemma 3): Suppose that for each $n,\left\{\mathbf{x}_{j}^{(n)}\right\}_{j=1}^{n}$ is a set of Gaussian random vectors with each $\mathbf{x}_{j}^{(n)}$ spherically symmetric in an $m_{j}(n)$-dimensional space. The variables may be dependent. Suppose also that $\mathbf{E}\left\|\mathbf{x}_{j}^{(n)}\right\|^{2}=1$ and $\lim _{n \rightarrow \infty} \log (n) / m_{\min }(n)=0$ where $m_{\min }(n)=\min _{j=1, \ldots, n} m_{j}(n)$. Then the limits

$$
\lim _{n \rightarrow \infty} \max _{j=1, \ldots, n}\left\|\mathbf{x}_{j}^{(n)}\right\|^{2}=\lim _{n \rightarrow \infty} \min _{j=1, \ldots, n}\left\|\mathbf{x}_{j}^{(n)}\right\|^{2}=1
$$

hold in probability.

Lemma 3 ([5], Lemma 4): Suppose that for each $n$, $\left\{u_{j}^{(n)}\right\}_{j=1}^{n}$ is a set of chi-squared random variables, each with one degree of freedom. The variables may be dependent. Then

$$
\limsup _{n \rightarrow \infty} \max _{j=1, \ldots, n} \frac{u_{j}^{(n)}}{2 \log (n)} \leq 1,
$$

where the limit is in probability.

The final two lemmas concern certain beta-distributed random variables. A real-valued scalar random variable $w$ follows a $\operatorname{Beta}(r, s)$ distribution if it can be written as $w=u_{r} /\left(u_{r}+v_{s}\right)$, where the variables $u_{r}$ and $v_{s}$ are independent chi-squared random variables with $r$ and $s$ degrees of freedom, respectively. The importance of the beta distribution is given by the following lemma.

Lemma 4 ([5, Lemma 5]): Suppose $\mathbf{x}$ and $\mathbf{y}$ are independent random $r$-dimensional random vectors with $\mathbf{x}$ being spherically-symmetrically distributed in $\mathbb{R}^{r}$ and $\mathbf{y}$ having any distribution that is nonzero with probability one. Then the random variable $w=\left|\mathbf{x}^{\prime} \mathbf{y}\right|^{2}\left(\|\mathbf{x}\|^{2}\|\mathbf{y}\|^{2}\right)$ is independent of $\mathbf{x}$ and follows a $\operatorname{Beta}(1, r-1)$ distribution.

The following lemma provides a simple expression for the maxima of certain beta-distributed variables. 
Lemma 5 ([5, Lemma 6]): For each $n$, suppose $\left\{w_{j}^{(n)}\right\}_{j=1}^{n}$ is a set of random variables with $w_{j}^{(n)}$ having a $\operatorname{Beta}\left(1, m_{j}(n)-1\right)$ distribution. Suppose that

$$
\lim _{n \rightarrow \infty} \log (n) / m_{\min }(n)=0, \quad \lim _{n \rightarrow \infty} m_{\min }(n)=\infty,
$$

where $m_{\min }(n)=\min _{j=1, \ldots, n} m_{j}(n)$. Then,

$$
\limsup _{n \rightarrow \infty} \max _{j=1, \ldots, n} \frac{m_{j}(n)}{2 \log (n)} w_{j}^{(n)} \leq 1
$$

in probability.

\section{Preliminary Computations and Technical Lemmas}

We first need to prove several simple but technical bounds. We begin by considering the dimension $m_{i}$ defined as

$$
m_{i}=\operatorname{dim}\left(\operatorname{range}\left(\mathbf{P}_{\text {true }}(i)\right)\right) \text {. }
$$

Our first lemma computes the limit of this dimension.

Lemma 6: The limit

$$
\lim _{n \rightarrow \infty} \min _{i=1, \ldots, n} \frac{m_{i}}{m-\lambda n}=1
$$

holds in probability and almost surely.

Proof: Recall that $\mathbf{P}_{\text {true }}(i)$ is the projection onto the orthogonal complement of the vectors $\mathbf{a}_{j}$ with $j \in I_{\text {true }}(i-1)$. With probability one, these vectors will be linearly independent, so $\mathbf{P}_{\text {true }}(i)$ will have dimension $m-\left|I_{\text {true }}(i-1)\right|$. Since $I_{\text {true }}(i)$ is increasing with $i$,

$$
\begin{aligned}
\min _{i=1, \ldots, n} m_{i} & =m-\max _{i=1, \ldots, n}\left|I_{\text {true }}(i-1)\right| \\
& =m-\left|I_{\text {true }}(n-1)\right| .
\end{aligned}
$$

Since each index is in the support with probability $\lambda$ and the $b_{j} \mathrm{~s}$ are independent, the law of large numbers shows that

$$
\lim _{n \rightarrow \infty} \frac{\left|I_{\text {true }}(n-1)\right|}{\lambda(n-1)}=1
$$

in probability and almost surely. Combining this with (42) and (23b) shows (41).

Next, for each $i=1, \ldots, n$, define the residual vector,

$$
\mathbf{e}_{i}=\mathbf{P}_{\text {true }}(i)\left(\mathbf{y}-\mathbf{a}_{i} x_{i}\right) .
$$

Observe that

$$
\begin{aligned}
\mathbf{e}_{i} & =\mathbf{P}_{\text {true }}(i)\left(\mathbf{y}-\mathbf{a}_{i} x_{i}\right) \stackrel{(a)}{=} \mathbf{P}_{\text {true }}(i)\left(\mathbf{d}+\sum_{j \neq i} \mathbf{a}_{j} x_{j}\right) \\
& \stackrel{(b)}{=} \mathbf{P}_{\text {true }}(i)\left(\mathbf{d}+\sum_{j>i} \mathbf{a}_{j} x_{j}\right),
\end{aligned}
$$

where (a) follows from (1) and (b) follows from the fact that $\mathbf{P}_{\text {true }}(i)$ is the projection onto the orthogonal complement of the span of all vectors $\mathbf{a}_{j}$ with $j<i$ and $x_{j} \neq 0$.

The next lemma shows that the power of the residual vector is described by the random variable

$$
\sigma^{2}(i)=1+\sum_{j=i+1}^{n}\left|x_{j}\right|^{2}
$$

Lemma 7: For all $i=1, \ldots, n$, the residual vector $\mathbf{e}_{i}$, conditioned on the modulation vector $\mathbf{x}$ and projection $\mathbf{P}_{\text {true }}(i)$, is a spherically symmetric Gaussian in the range space of $\mathbf{P}_{\text {true }}(i)$ with total variance

$$
\mathbf{E}\left(\left\|\mathbf{e}_{i}\right\|^{2} \mid \mathbf{x}\right)=\frac{m_{i}}{m} \sigma^{2}(i),
$$

where $m_{i}$ and $\sigma^{2}(i)$ are defined in (40) and (44), respectively.

Proof: Let $\mathbf{v}_{i}=\mathbf{d}+\sum_{j>i} \mathbf{a}_{j} x_{j}$, so that $\mathbf{e}_{i}=\mathbf{P}_{\text {true }}(i) \mathbf{v}_{i}$. Since the vectors $\mathbf{a}_{j}$ and $\mathbf{d}$ have Gaussian $\mathcal{N}\left(0,1 / m I_{m}\right)$ distributions, for a given vector $\mathbf{x}, \mathbf{v}_{i}$ must be a zero-mean white Gaussian vector with total variance $\mathbf{E}\left\|\mathbf{v}_{i}\right\|^{2}=\sigma^{2}(i)$. Also, since the operator $\mathbf{P}_{\text {true }}(i)$ is a function of the components $x_{\ell}$ and vectors $\mathbf{a}_{\ell}$ for $\ell<i, \mathbf{P}_{\text {true }}(i)$ is independent of the vectors $\mathbf{d}$ and $\mathbf{a}_{j}, j>i$, and therefore independent of $\mathbf{v}_{i}$. Since $\mathbf{P}_{\text {true }}(i)$ is a projection from an $m$-dimensional space to an $m_{i}$-dimensional space, $\mathbf{e}_{i}$, conditioned on the modulation vector $\mathbf{x}$, must be spherically symmetric Gaussian in the range space of $\mathbf{P}_{\text {true }}(i)$ with total variance satisfying (45).

Our next lemma requires the following version of the wellknown Hoeffding's inequality.

Lemma 8 (Hoeffding's Inequality): Suppose $z$ is the $\operatorname{sum} z=$ $z_{0}+\sum_{i=1}^{r} z_{i}$ where $z_{0}$ is a constant and the variables $z_{i}$ are independent random variables that are almost surely bounded in some interval $z_{i} \in\left[a_{i}, b_{i}\right]$. Then, for all $\epsilon>0$,

$$
\operatorname{Pr}(z-\mathbf{E}(z) \geq \epsilon) \leq \exp \left(\frac{-2 \epsilon^{2}}{C}\right),
$$

where $C=\sum_{i=1}^{r}\left(b_{i}-a_{i}\right)^{2}$.

Proof: See [45].

Lemma 9: Under the assumptions of Theorem 1, the limit

$$
\limsup _{n \rightarrow \infty} \max _{i=1, \ldots, n} \frac{\sigma^{2}(i)}{\hat{\sigma}^{2}(i)} \leq 1
$$

holds in probability.

Proof: Let $z(i)=\sigma^{2}(i) / \hat{\sigma}^{2}(i)$. From the definition of $\sigma^{2}(i)$ in (44), we can write

$$
z(i)=\frac{1}{\hat{\sigma}^{2}(i)}+\sum_{j=i+1}^{n} z(i, j),
$$

where $z(i, j)=\left|x_{j}\right|^{2} / \hat{\sigma}^{2}(i)$ for $j>i$.

Now recall that in the problem formulation, each $x_{j}$ is nonzero with probability $\lambda$, with conditional power $p_{j}$. Also, the activity variables $\left\{b_{j}\right\}_{j=1}^{n}$ are independent, and the conditional powers $p_{j}$ are deterministic quantities. Therefore, the variables $z(i, j)$ are independent with

$$
z(i, j)= \begin{cases}p_{j} / \hat{\sigma}^{2}(i), & \text { with probability } \lambda \\ 0, & \text { with probability } 1-\lambda,\end{cases}
$$

for $j>i$. Combining this with the definition of $\hat{\sigma}^{2}(i)$ in (22b), we see that

$$
\mathbf{E}(z(i))=\frac{1}{\hat{\sigma}^{2}(i)}\left(1+\lambda \sum_{j=i+1}^{n} p_{j}\right)=1 .
$$

Also, for each $j>i$, we have the bound $z(i, j) \in\left[0, p_{j} / \hat{\sigma}^{2}(i)\right]$. So for use in Hoeffding's Inequality (Lemma 8), define

$$
C=C(i, n)=\hat{\sigma}^{-4}(i) \sum_{j=i+1}^{n} p_{j}^{2},
$$


where dependence of the power profile and $\hat{\sigma}(i)$ on $n$ is implicit. Now define

$$
c_{n}=\max _{i=1, \ldots, n} \log (n) C(i, n)
$$

so that $C(i, n) \leq c_{n} / \log (n)$ for all $i$. Hoeffding's Inequality (Lemma 8) now shows that for all $i<n$,

$$
\begin{aligned}
\operatorname{Pr}(z(i) \geq 1+\epsilon) & \leq \exp \left(-2 \epsilon^{2} / C(i, n)\right) \\
& \leq \exp \left(-2 \epsilon^{2} \log (n) / c_{n}\right) .
\end{aligned}
$$

Using the union bound,

$$
\begin{gathered}
\lim _{n \rightarrow \infty} \operatorname{Pr}\left(\max _{j=1, \ldots, n} z(i)>1+\epsilon\right) \\
\leq \lim _{n \rightarrow \infty} n \exp \left(-\frac{2 \epsilon^{2} \log (n)}{c_{n}}\right) \\
\quad=\lim _{n \rightarrow \infty} n^{1-2 \epsilon^{2} / c_{n}}=0 .
\end{gathered}
$$

The final step is due to the fact that the technical condition (23c) in the theorem implies $c_{n} \rightarrow 0$. This proves the lemma.

\section{Missed Detection Probability}

Consider any $j \in I_{\text {true }}$. Using (43) to rewrite (36) along with some algebra shows

$$
\begin{aligned}
\rho_{\text {true }}(j) & =\frac{\left|\mathbf{a}_{j}^{\prime} \mathbf{P}_{\text {true }}(j) \mathbf{y}\right|^{2}}{\left\|\mathbf{P}_{\text {true }}(j) \mathbf{a}\right\|^{2}\left\|\mathbf{P}_{\text {true }}(j) \mathbf{y}_{j}\right\|^{2}} \\
& =\frac{\left|\mathbf{a}_{j}^{\prime}\left(x_{j} \mathbf{P}_{\text {true }}(j) \mathbf{a}_{j}+\mathbf{e}_{j}\right)\right|^{2}}{\left\|\mathbf{P}_{\text {true }}(j) \mathbf{a}_{j}\right\|^{2}\left\|x_{j} \mathbf{P}_{\text {true }}(j) \mathbf{a}_{j}+\mathbf{e}_{j}\right\|^{2}} \\
& \geq \frac{s_{j}-2 \sqrt{z_{j} s_{j}}+z_{j}}{s_{j}+2 \sqrt{z_{j} s_{j}}+1},
\end{aligned}
$$

where

$$
\begin{aligned}
s_{j} & =\frac{\left|x_{j}\right|^{2}\left\|\mathbf{P}_{\text {true }}(j) \mathbf{a}_{j}\right\|^{2}}{\left\|\mathbf{e}_{j}\right\|^{2}}, \\
z_{j} & =\frac{\left|\mathbf{a}_{j}^{\prime} \mathbf{P}_{\text {true }}(j) \mathbf{e}_{j}\right|^{2}}{\left\|\mathbf{P}_{\text {true }}(j) \mathbf{a}_{j}\right\|^{2}\left\|\mathbf{e}_{j}\right\|^{2}} .
\end{aligned}
$$

Define

$$
s_{\min }=\min _{j \in I_{\text {true }}} s_{j}, \quad s_{\max }=\max _{j \in I_{\text {true }}} z_{j} .
$$

We will now bound $s_{\min }$ from below and $s_{\max }$ from above.

We first start with $s_{\text {min }}$. Conditional on $\mathbf{x}$ and $\mathbf{P}_{\text {true }}(j)$, Lemma 7 shows that each $\mathbf{e}_{j}$ is a spherically-symmetrically distributed Gaussian on the $m_{j}$-dimensional range space of $\mathbf{P}_{\text {true }}(j)$. Since there are asymptotically $\lambda n$ elements in $I_{\text {true }}$, Lemma 2 along with (23b) show that

$$
\lim _{n \rightarrow \infty} \max _{j \in I_{\text {true }}} \frac{m}{m_{j} \sigma^{2}(j)}\left\|\mathbf{e}_{j}\right\|^{2}=1,
$$

where the limit is in probability. Similarly, $\mathbf{P}_{\text {true }}(j) \mathbf{a}_{j}$ is also a spherically-symmetrically distributed Gaussian in the range space of $\mathbf{P}_{\text {true }}(j)$. Since $\mathbf{P}_{\text {true }}(j)$ is a projection from an $m$-dimensional space to a $m_{j}$-dimensional space and $\mathbf{E}\left\|\mathbf{a}_{j}\right\|^{2}=1$, we have that $\mathbf{E}\left\|\mathbf{P}_{\text {true }}(j) \mathbf{a}_{j}\right\|^{2}=m_{j} / m$. Therefore, Lemma 2 along with (23b) show that

$$
\lim _{n \rightarrow \infty} \min _{j \in I_{\text {true }}} \frac{m}{m_{j}}\left\|\mathbf{P}_{\text {true }}(j) \mathbf{e}_{j}\right\|^{2}=1 .
$$

Taking the limit (in probability) of $s_{\min }$,

$$
\begin{aligned}
\liminf _{n \rightarrow \infty} \frac{s_{\text {min }}}{\gamma} & =\liminf _{n \rightarrow \infty} \min _{j \in I_{\text {true }}} \frac{s_{j}}{\gamma} \\
& \stackrel{(a)}{=} \liminf _{n \rightarrow \infty} \min _{j \in I_{\text {true }}} \frac{\left|x_{j}\right|^{2}\left\|\mathbf{P}_{\text {true }}(j) \mathbf{a}_{j}\right\|^{2}}{\gamma\left\|\mathbf{e}_{j}\right\|^{2}} \\
& \stackrel{(b)}{=} \liminf _{n \rightarrow \infty} \min _{j \in I_{\text {true }}} \frac{\left|x_{j}\right|^{2}}{\gamma \sigma^{2}(j)} \\
& \stackrel{(c)}{=} \liminf _{n \rightarrow \infty} \min _{j \in I_{\text {true }}} \frac{p_{j}}{\gamma \sigma^{2}(j)} \\
& \stackrel{(d)}{\geq} \liminf _{n \rightarrow \infty} \min _{j \in I_{\text {true }}} \frac{p_{j}}{\gamma \hat{\sigma}^{2}(j)} \stackrel{(e)}{\geq} 1
\end{aligned}
$$

where (a) follows from (47); (b) follows from (49) and (50); (c) follows from (20); (d) follows from Lemma 9; and (e) follows from (22a).

We next consider $s_{\text {max }}$. Conditional on $\mathbf{P}_{\text {true }}(j)$, the vectors $\mathbf{P}_{\text {true }}(j) \mathbf{a}_{j}$ and $\mathbf{e}_{j}$ are independent spherically-symmetric Gaussians in the range space of $\mathbf{P}_{\text {true }}(j)$. It follows from Lemma 4 that each $z_{j}$ is a $\operatorname{Beta}\left(1, m_{j}-1\right)$ random variable. Since there are asymptotically $\lambda n$ elements in $I_{\text {true }}$, Lemma 5 along with (41) and (23b) show that

$$
\limsup _{n \rightarrow \infty} \frac{m-\lambda n}{2 \log (\lambda n)} s_{\max }=\limsup _{n \rightarrow \infty} \frac{m-\lambda n}{2 \log (\lambda n)} \max _{j \in I_{\text {true }}} z_{j} \leq 1 .
$$

The above analysis shows that for any $j \in I_{\text {true }}$,

$$
\begin{aligned}
& \liminf _{n \rightarrow \infty} \min _{j \in I_{\text {true }}} \frac{1}{\sqrt{\mu}}\left(\sqrt{s_{j}}-\sqrt{z_{j}}\right) \\
& \quad \stackrel{(a)}{\geq} \liminf _{n \rightarrow \infty} \frac{1}{\sqrt{\mu}}\left(\sqrt{s_{\min }}-\sqrt{s_{\max }}\right) \\
& \quad \stackrel{(b)}{\geq} \liminf _{n \rightarrow \infty} \frac{1}{\sqrt{\mu}}\left(\sqrt{\gamma}-\sqrt{\frac{2 \log (\lambda n)}{m-\lambda n}}\right) \\
& \geq \liminf _{n \rightarrow \infty} \sqrt{\frac{1+\delta}{\mu}\left(\sqrt{\frac{\gamma}{1+\delta}}-\sqrt{\frac{2 \log (\lambda n)}{m-\lambda n}}\right)} \\
& \quad \stackrel{(c)}{\geq} \liminf _{n \rightarrow \infty} \sqrt{\frac{2(1+\delta)}{(m-\lambda n) \mu}} \\
& \quad((1+\sqrt{\gamma}) \sqrt{L(\lambda n, n)}-\sqrt{\log (\lambda n)}) \\
& \stackrel{(d)}{=} \liminf _{n \rightarrow \infty}(1+\sqrt{\gamma}) \sqrt{\frac{2(1+\delta) \log (n(1-\lambda))}{(m-\lambda n) \mu}} \\
& \stackrel{(e)}{=} \liminf _{n \rightarrow \infty}(1+\sqrt{\gamma}) \sqrt{\frac{1+\delta}{1+\epsilon}} \\
& \stackrel{(f)}{\geq}(1+\sqrt{\gamma}) \sqrt{1+\epsilon}
\end{aligned}
$$

where (a) follows from the definitions of $s_{\min }$ and $s_{\max }$; (b) follows from (51) and (52); (c) follows from (24); (d) follows from 
(14); (e) follows from (39); and (f) follows from (38). Therefore, starting with (46),

$$
\begin{aligned}
& \liminf _{n \rightarrow \infty} \min _{j \in I_{\text {true }}} \frac{\rho(j)}{\mu} \\
& \quad \stackrel{(a)}{\geq} \liminf _{n \rightarrow \infty} \min _{j \in I_{\text {true }}} \frac{1}{\mu} \cdot \frac{s_{j}-2 \sqrt{z_{j} s_{j}}+z_{j}}{s_{j}+2 \sqrt{z_{j} s_{j}}+1} \\
& \quad=\liminf _{n \rightarrow \infty} \min _{j \in I_{\text {true }}} \frac{1}{\mu} \cdot \frac{\left(\sqrt{s_{j}}-\sqrt{z_{j}}\right)^{2}}{s_{j}+2 \sqrt{z_{j} s_{j}}+1} \\
& \quad \stackrel{(b)}{\geq} \liminf _{n \rightarrow \infty} \min _{j \in I_{\text {true }}} \frac{(1+\sqrt{\gamma})^{2}(1+\epsilon)}{s_{j}+2 \sqrt{z_{j} s_{j}}+1} \\
& \quad \stackrel{(c)}{\geq} \liminf _{n \rightarrow \infty} \min _{j \in I_{\text {true }}} \frac{(1+\sqrt{\gamma})^{2}(1+\epsilon)}{s_{j}+2 \sqrt{s_{j}}+1} \\
& \quad \geq \liminf _{n \rightarrow \infty} \min _{j \in I_{\text {true }}} \frac{(1+\sqrt{\gamma})^{2}(1+\epsilon)}{s_{\text {min }}+2 \sqrt{s_{\min }}+1} \\
& \quad \stackrel{(d)}{\geq} \liminf _{n \rightarrow \infty} \min _{j \in I_{\text {true }}} \frac{(1+\sqrt{\gamma})^{2}(1+\epsilon)}{(1+\sqrt{\gamma})^{2}}=1+\epsilon,
\end{aligned}
$$

where (a) follows from (46); (b) follows from (53); (c) follows from the fact that $z_{j} \in[0,1]$ (it is a beta-distributed random variable); (d) follows from (51). This proves the first requirement, condition (37a).

\section{E. False Alarm Probability}

Now consider any index $j \notin I_{\text {true }}$. This implies that $x_{j}=0$ and therefore (43) shows that

$$
\mathbf{P}_{\text {true }}(j) \mathbf{y}=\mathbf{e}_{j} .
$$

Hence from (36),

$$
\rho_{\text {true }}(j)=\frac{\left|\mathbf{a}_{j}^{\prime} \mathbf{e}\right|^{2}}{\left\|\mathbf{P}_{\text {true }}(j) \mathbf{a}\right\|^{2}\left\|\mathbf{e}_{j}\right\|^{2}}=z_{j}
$$

where $z_{j}$ is defined in (48). From the discussion above, each $z_{j}$ has the $\operatorname{Beta}\left(1, m_{j}-1\right)$ distribution. Since there are asymptotically $(1-\lambda) n$ elements in $I_{\text {true }}^{c}$, the conditions (41) and (23b) along with Lemma 5 show that the limit

$$
\limsup _{n \rightarrow \infty} \max _{j \notin I_{\text {true }}} \frac{m-\lambda n}{2 \log (n(1-\lambda))} z_{j} \leq 1
$$

holds in probability. Therefore,

$$
\begin{aligned}
& \underset{\substack{n \rightarrow \infty \\
\limsup }}{ } \max _{j \notin I_{\text {true }}} \frac{1}{\mu} \rho_{\text {true }}(j) \\
& \quad \stackrel{(a)}{=} \limsup _{n \rightarrow \infty} \max _{j \notin I_{\text {true }}} \frac{1}{\mu} z_{j} \\
& \quad \stackrel{(b)}{=} \limsup _{n \rightarrow \infty} \max _{j \notin I_{\text {true }}} \frac{m-\lambda n}{(1+\epsilon)(1+\sqrt{\gamma})^{2} 2 \log (n(1-\lambda))} z_{j} \\
& \quad \stackrel{(c)}{\leq} \frac{1}{1+\epsilon}
\end{aligned}
$$

where (a) follows from (54); (b) follows from (39); and (c) follows from (55). This proves (37b) and thus completes the proof of the theorem.

\section{ACKNOWLEDGMENT}

The authors thank Martin Vetterli for his support, wisdom, and encouragement. The authors also thank Gerhard Kramer for helpful comments on an early draft and the anonymous reviewers and Associate Editor for several useful suggestions.

\section{REFERENCES}

[1] A. K. Fletcher, S. Rangan, and V. K Goyal, "A sparsity detection framework for on-off random access channels," in Proc. IEEE Int. Symp. Inf Theory, Seoul, Korea, Jun.-Jul. 2009, pp. 169-173.

[2] S. Mallat, $A$ Wavelet Tour of Signal Processing, 2nd ed. New York: Academic, 1999.

[3] M. J. Wainwright, "Information-theoretic limits on sparsity recovery in the high-dimensional and noisy setting," IEEE Trans. Inf. Theory, vol. 55 , no. 12 , pp. $5728-5741$, Dec. 2009

[4] M. J. Wainwright, "Sharp thresholds for high-dimensional and noisy sparsity recovery using $\ell_{1}$-constrained quadratic programming (Lasso)," IEEE Trans. Inf. Theory, vol. 55, no. 5, pp. 2183-2202, May 2009.

[5] A. K. Fletcher, S. Rangan, and V. K Goyal, "Necessary and sufficient conditions for sparsity pattern recovery," IEEE Trans. Inf. Theory, vol. 55, no. 12 , pp. 5758-5772, Dec. 2009.

[6] B. K. Natarajan, "Sparse approximate solutions to linear systems," SIAM J. Comput., vol. 24, no. 2, pp. 227-234, Apr. 1995.

[7] S. G. Mallat and Z. Zhang, "Matching pursuits with time-frequency dictionaries," IEEE Trans. Signal Process., vol. 41, no. 12, pp. 3397-3415, Dec. 1993

[8] S. Chen, S. A. Billings, and W. Luo, "Orthogonal least squares methods and their application to non-linear system identification," Int. J. Control, vol. 50, no. 5, pp. 1873-1896, Nov. 1989

[9] Y.C. Pati, R. Rezaiifar, and P. S. Krishnaprasad, "Orthogonal matching pursuit: Recursive function approximation with applications to wavelet decomposition," in Proc. Conf. Rec. 27th Asilomar Conf. Signals, Syst., Comput., Pacific Grove, CA, Nov. 1993, vol. 1, pp. 40-44.

[10] G. Davis, S. Mallat, and Z. Zhang, "Adaptive time-frequency decomposition," Opt. Eng., vol. 33, no. 7, pp. 2183-2191, Jul. 1994.

[11] D. Needell and J. A. Tropp, "CoSaMP: Iterative signal recovery from incomplete and inaccurate samples," Appl. Comput. Harm. Anal., vol. 26, no. 3, pp. 301-321, May 2009.

[12] W. Dai and O. Milenkovic, "Subspace pursuit for compressive sensing signal reconstruction," IEEE Trans. Inf. Theory, vol. 55, no. 5, pp. 2230-2249, May 2009

[13] S. S. Chen, D. L. Donoho, and M. A. Saunders, "Atomic decomposition by basis pursuit" SIAM J. Sci. Comp., vol. 20, no. 1, pp. 33-61, 1999.

[14] R. Tibshirani, "Regression shrinkage and selection via the lasso," $J$ Royal Stat. Soc., Ser. B, vol. 58, no. 1, pp. 267-288, 1996.

[15] E. J. Candès and T. Tao, "The Dantzig selector: Statistical estimation when $p$ is much larger than $n$," Ann. Stat., vol. 35, no. 6, pp. 2313-2351, Dec. 2007.

[16] D. L. Donoho, M. Elad, and V. N. Temlyakov, "Stable recovery of sparse overcomplete representations in the presence of noise," IEEE Trans. Inf. Theory, vol. 52, no. 1, pp. 6-18, Jan. 2006.

[17] J. A. Tropp, "Greed is good: Algorithmic results for sparse approximation," IEEE Trans. Inf. Theory, vol. 50, no. 10, pp. 2231-2242, Oct. 2004.

[18] J. A. Tropp, "Just relax: Convex programming methods for identifying sparse signals in noise," IEEE Trans. Inf. Theory, vol. 52, no. 3, pp. 1030-1051, Mar. 2006.

[19] E. J. Candès, J. Romberg, and T. Tao, "Robust uncertainty principles: Exact signal reconstruction from highly incomplete frequency information," IEEE Trans. Inf. Theory, vol. 52, no. 2, pp. 489-509, Feb. 2006.

[20] D. L. Donoho, "Compressed sensing," IEEE Trans. Inf. Theory, vol. 52, no. 4, pp. 1289-1306, Apr. 2006.

[21] E. J. Candès and T. Tao, "Near-optimal signal recovery from random projections: Universal encoding strategies?," IEEE Trans. Inf. Theory, vol. 52 , no. 12 , pp. $5406-5425$, Dec. 2006.

[22] S. Rangan, A. Fletcher, and V. K Goyal, "Asymptotic analysis of MAP estimation via the replica method and applications to compressed sensing," IEEE Trans. Inf. Theory, vol. 58, no. 3, pp. 1902-1923, Mar. 2012.

[23] D. L. Donoho and J. Tanner, "Counting faces of randomly-projected polytopes when the projection radically lowers dimension," J. Amer. Math. Soc., vol. 22, no. 1, pp. 1-53, Jan. 2009.

[24] S. Sarvotham, D. Baron, and R. G. Baraniuk, "Measurements vs. bits: Compressed sensing meets information theory," in Proc. 44th Ann. Allerton Conf. Commun., Contr., Comp., Monticello, IL, Sep. 2006.

[25] A. K. Fletcher, S. Rangan, and V. K Goyal, "Rate-distortion bounds for sparse approximation," in Proc. IEEE Statist. Signal Process. Workshop, Madison, WI, Aug. 2007, pp. 254-258.

[26] G. Reeves, "Sparse signal sampling using noisy linear projections," Univ. of Calif., Dep. Elec. Eng. and Comp. Sci., Berkeley, Tech. Rep. UCB/EECS-2008-3, Jan. 2008.

[27] M. Akçakaya and V. Tarokh, "Shannon-theoretic limits on noisy compressive sampling," IEEE Trans. Inf. Theory, vol. 56, no. 1, pp. 492-504, Jan. 2010

[28] J. A. Tropp and A. C. Gilbert, "Signal recovery from random measurements via orthogonal matching pursuit," IEEE Trans. Inf. Theory, vol. 53, no. 12, pp. 4655-4666, Dec. 2007. 
[29] A. K. Fletcher and S. Rangan, "Orthogonal matching pursuit: A Brownian motion analysis," IEEE Trans. Signal Process., vol. 60, no. 3, pp. 1010-1021, Mar. 2012.

[30] H. Rauhut, K. Schnass, and P. Vandergheynst, "Compressed sensing and redundant dictionaries," IEEE Trans. Inf. Theory, vol. 54, no. 5, pp. 2210-2219, May 2008.

[31] R. G. Baraniuk, V. Cevher, M. F. Duarte, and C. Hegde, "Model-based compressed sensing," IEEE Trans. Inf. Theory, vol. 56, no. 4, pp. 1982-2001, Apr. 2010.

[32] N. Vaswani and W. Lu, "Modified-CS: Modifying compressive sensing for problems with partially known support," IEEE Trans. Signal Process., vol. 58, no. 9, pp. 4595-4607, Sep. 2010.

[33] M. A. Khajehnejad, W. Xu, A. S. Avestimehr, and B. Hassibi, "Analyzing weighted $\ell_{1}$ minimization for sparse recovery with nonuniform sparse models," IEEE Trans. Signal Process., vol. 59, no. 5, pp. 1985-2001, May 2011.

[34] S. Rangan, A. K. Fletcher, V. K Goyal, and P. Schniter, "Hybrid approximate message passing with applications to structured sparsity," Nov. 2011 [Online]. Available: http://arxiv.org/abs/1111.2581

[35] S. Rangan, "Generalized approximate message passing for estimation with random linear mixing," Oct. 2010 [Online]. Available: http://arxiv.org/abs/1010.5141

[36] W. Wang, M. J. Wainwright, and K. Ramchandran, "Information-theoretic limits on sparse signal recovery: Dense versus sparse measurement matrices," IEEE Trans. Inf. Theory, vol. 56, no. 6, pp. 2967-2979, Jun. 2010

[37] M. F. Duarte, S. Sarvotham, D. Baron, W. B. Wakin, and R. G. Baraniuk, "Distributed compressed sensing of jointly sparse signals," in Proc. Conf. Rec. Asilomar Conf. Signals, Syst. Comput., Pacific Grove, CA, Oct.-Nov. 2005, pp. 1537-1541.

[38] M. Tipping, "Sparse Bayesian learning and the relevance vector machine," J. Mach. Learn. Res., vol. 1, pp. 211-244, Sep. 2001.

[39] D. Wipf and B. Rao, "Sparse Bayesian learning for basis selection," IEEE Trans. Signal Process., vol. 52, no. 8, pp. 2153-2164, Aug. 2004

[40] P. Schniter, L. C. Potter, and J. Ziniel, "Fast Bayesian matching pursuit: Model uncertainty and parameter estimation for sparse linear models," IEEE Trans. Signal Process., Aug. 2008, submitted for publication.

[41] D. Wipf and B. Rao, "Comparing the effects of different weight distributions on finding sparse representations," presented at the Proc. Neur. Inf. Process. Syst., Vancouver, Canada, Dec. 2006.

[42] A. Agrawal, J. G. Andrews, J. M. Cioffi, and T. Meng, "Iterative power control for imperfect successive interference cancellation," IEEE Trans. Wireless Commun., vol. 4, no. 3, pp. 878-884, May 2005.

[43] A. K. Fletcher, S. Rangan, and V. K Goyal, "Ranked sparse signal support detection," Oct. 2011 [Online]. Available: http://arxiv.org/abs/ 1110.6188

[44] M. Evans, N. Hastings, and J. B. Peacock, Statistical Distributions, 3rd ed. New York: Wiley, 2000.

[45] W. Hoeffding, "Probability inequalities for sums of bounded random variables," J. Amer. Stat. Assoc., vol. 58, no. 301, pp. 13-30, Mar. 1963.

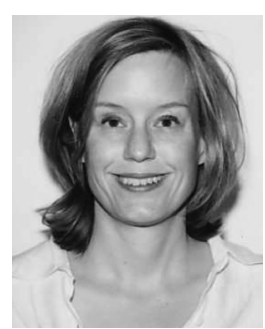

Alyson K. Fletcher (S'03-M'04) received the B.S. degree in mathematics from the University of Iowa, Iowa City. She received the M.S. degree in electrical engineering in 2002, the M.A. degree in mathematics, and the Ph.D. degree in electrical engineering, both in 2006, all from the from the University of California, Berkeley.

She is an Assistant Professor of Electrical Engineering in the Jack Baskin School of Engineering, University of California at Santa Cruz. Her research interests include signal processing, information theory, machine learning, and neuroscience.
Dr. Fletcher is a member of SWE, SIAM, and Sigma Xi. In 2005, she received the University of California Eugene L. Lawler Award, the Henry Luce Foundations Clare Boothe Luce Fellowship, the Soroptimist Dissertation Fellowship, and University of California President's Postdoctoral Fellowship.

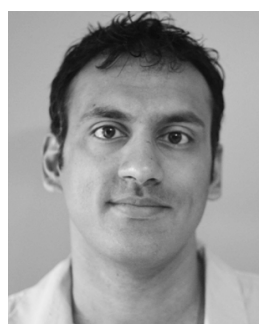

Sundeep Rangan (M'02) received the B.A.Sc. degree from the University of Waterloo, Canada, and the M.S. and Ph.D. degrees from the University of California, Berkeley, all in electrical engineering.

$\mathrm{He}$ held postdoctoral appointments with the University of Michigan, Ann Arbor, and Bell Labs. In 2000, he cofounded (with four others) Flarion Technologies, a spin-off of Bell Labs, that developed Flash OFDM, one of the first cellular OFDM data systems. In 2006, Flarion was acquired by Qualcomm Technologies, where he was a Director of Engineering involved in OFDM infrastructure products. He joined the Department of Electrical and Computer Engineering, Polytechnic Institute of New York University, in 2010, where he is currently an Associate Professor. His research interests are in wireless communications, signal processing, information theory, and control theory.

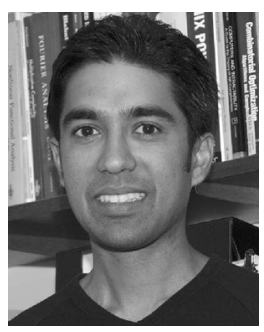

Vivek K Goyal (S'92-M'98-SM'03) received the B.S. degree in mathematics and the B.S.E. degree in electrical engineering from the University of Iowa, Iowa City, where he received the John Briggs Memorial Award for the top undergraduate across all colleges. He received the M.S. and Ph.D. degrees in electrical engineering from the University of California, Berkeley, where he received the Eliahu Jury Award for outstanding achievement in systems, communications, control, or signal processing.

He was a Member of Technical Staff in the Mathematics of Communications Research Department of Bell Laboratories, Lucent Technologies, from 1998 to 2001; and a Senior Research Engineer for Digital Fountain, Inc., during 2001-2003. He has been with the Massachusetts Institute of Technology, Cambridge, since 2004. His research interests include computational imaging, sampling, quantization, and source coding theory.

Dr. Goyal is a member of Phi Beta Kappa, Tau Beta Pi, Sigma Xi, Eta Kappa $\mathrm{Nu}$, and SIAM. He was awarded the 2002 IEEE Signal Processing Society Magazine Award and an NSF CAREER Award. As a research supervisor, he is coauthor of papers that won Student Best Paper awards at the IEEE Data Compression Conference in 2006 and 2011 and the IEEE Sensor Array and Multichannel Signal Processing Workshop in 2012. He served on the IEEE Signal Processing Society's Image and Multiple Dimensional Signal Processing Technical Committee from 2003 to 2009. He is a Technical Program Committee Co-Chair of IEEE ICIP 2016 and a permanent Conference Co-Chair of the SPIE Wavelets and Sparsity conference series. 